\title{
MiR-148a-3p suppresses cell proliferation, migration and invasion by targeting PIK3CA in human osteosarcoma cells
}

\author{
Xu-Ke Wang ${ }^{1}$, Ying-Jie Zhou ${ }^{1}$, Gang Zhao ${ }^{1}$ and Huai-Liang Zheng ${ }^{1}$ \\ ${ }^{1}$ Department of Orthopedics, Henan Provincial Luoyang Orthopedic-Traumatological Hospital (Henan Provincial Orthopedic \\ Hospital), Luoyang, 471002, Henan, China
}

Correspondence to: Ying-Jie Zhou, email: zhouyj017@163.com

Keywords: miR-148a-3p; PIK3CA; osteosarcoma; proliferation; invasion

Received: August 31, $2017 \quad$ Accepted: November 03, $2017 \quad$ Published: January 03, 2018

Copyright: Wang et al. This is an open-access article distributed under the terms of the Creative Commons Attribution License 3.0 (CC BY 3.0), which permits unrestricted use, distribution, and reproduction in any medium, provided the original author and source are credited.

\begin{abstract}
Osteosarcoma (OS) is the most common type of primary malignant bone tumor and is characterized by complex genetic changes and resistance to conventional treatments. In this study, the role of $\mathrm{miR-148a-3p}$ in the progression and metastasis of OS was investigated. Firstly, it was found that the expression of miR-148a-3p was down-regulated in OS tissues and cell lines compared with that in adjacent non-neoplastic bone tissues and OS cell line. In addition, it was found that miR148a-3p inhibited cell proliferation, migration and invasion in MG-63 and U2OS cells and tumorigenicity in nude mice. Meanwhile, bioinformatic analysis suggested that phosphatidylinositol-4, 5-bisphosphate 3-kinase, catalytic subunit alpha (PIK3CA) gene is a potential target of miR-148a-3p. Then, it was found that PIK3CA can be directly regulated by $\mathrm{miR-148a-3p}$ via luciferase assay and western blot assay. Furthermore, PIK3CA knockdown induced effects on OS cell lines similar to those induced by miR-148a-3p. Taken together, these data suggest that miR-148a-3p suppresses cell proliferation, migration and invasion and PIK3CA was involved in miR-148a-3p associated biological functions in OS. Commonly down-regulated miR148a-3p may act as a tumor suppressor and a novel therapeutic strategy by downregulating PIK3CA expression.
\end{abstract}

\section{INTRODUCTION}

Osteosarcoma (OS) is the most common malignant bone tumor in children and adolescents [1]. With the combination of surgery and other multiagent chemotherapy, the 5-year cumulative survival rate of primary OS has improved nearly to $80 \%$, the prognosis for OS remains poor $[2,3]$. The mechanisms of formation and development of OS remain poorly understood [4]. Recently, increasing evidences showed that miRNAs play important roles in regulating tumor growth [5-7].

MiRNAs are a class of small, noncoding RNA molecules that post-transcriptionally regulate target mRNAs [8]. Deregulated miRNAs are found in lots of diseases including tumors, where they can either serve as tumor oncogenes or suppressors, or regulate various tumor oncogenes and suppressors [9, 10], playing roles in pathological and physiological processes such as cell-cycle regulation, differentiation, proliferation, apoptosis and migration [11-14]. Recently, the roles of miRNAs and their targets in the initiation and progression of OS have been reported, Such as miR-15a, miR-203, miR-218, miR-375, miR-449, miR-539, miR-542-5p and miR-646 [15-22].

In this study, the roles of miR-148a-3p in OS were investigated. We found that miR-148a-3p was downregulated in OS cells and tumor bone tissues. Moreover, it was demonstrated that miR-148a-3p inhibited cell proliferation, migration and invasion in OS cells. PIK3CA was identified as a novel target of miR-148a-3p and involved in suppressive effect of miR-148a-3p in OS.

\section{RESULTS}

MiR-148a-3p is downregulated in OS tissues and cell lines

Firstly, we measured the expression of miR-148a$3 p$ in 56 pairs of OS tumor versus adjacent non-tumor 
tissues by qRT-PCR. MiR-148a-3p was downregulated in tumor tissues compared to the non-tumor tissues tested (Figure 1A). In addition, we measured the expression of miR-148a-3p in three human OS cells lines (MG63 , U2OS and Saos2) and in human osteoblast cell line hFOB1.19 by qRT-PCR. As expected, the expression of miR-148a-3p was much lower in three OS cell lines than that in hFOB1.19 (Figure 1B).

\section{MiR-148a-3p inhibits OS cell proliferation and tumorigenicity}

MG-63 and U2OS cells were transfected with miR148a-3p mimic, inhibitor or NC duplex. Firstly, qRT-PCT results confirmed miR-148a-3p expression level was significant elevated by miR-148a-3p mimic transfection (Figure 2A). Then, it was showed that cell viability was decreased in miR-148a-3p mimic transfected MG-63 and U2OS cells compared with either the NC duplex transfected cells or the untreated cells (Figure 2B). Conversely, MG-63 and U2OS cells transfected with miR148a-3p inhibitor showed higher cell proliferation capacity (Figure 2B). Thus, miR-148a-3p significantly suppressed the cell proliferation in OS cells.

To validate the inhibitory effect of miR-148a-3p on cell proliferation, the colony formation assay was performed in MG-63 and U2OS cells transfected with miR-148a-3p mimic, miR-148a-3p inhibitor or NC duplex or not. Similarly, MG-63 and U2OS cells transfected with miR-148a-3p mimic displayed much fewer and smaller colonies (459 or 762 colonies) than those of NC duplex transfected (1083 or 1819 colonies) and nontransfected cells (1058 or 1761 colonies), but miR-148a-3p inhibitor transfected cells displayed much more and larger colonies (1764 or 2208 colonies)(Figure 2C).

Furthermore, cell tumorigenicity assay in nude mouse model was performed. During the treatment with miR-148a-3p mimic or miR-148a-3p inhibitor for 5 weeks, growth rates showed a significant decrease at the 4 th and 5th week but a significant increase at the 5th week, whereas no significance were observed in NC group or the ctrl group (Figure 2D). These results indicated that introduction of miR-148a-3p inhibits tumorigenicity of MG-63 cells significantly in vivo.

\section{MiR-148a-3p inhibits OS cell migration and invasion}

Firstly, Transwell migration and invasion were assayed in OS MG-63 and U2OS cells. MiR-148a-3p mimic transfected cells were distinctively less migratory than NC transfected cells or untreated cells. However, miR-148a-3p inhibitor transfected cells showed much more migratory (Figure 3A). Furthermore, Matrigel cell invasion assay was conducted and then the invaded cells were stained. The number of invaded cells transfected with the miR-148a-3p mimic was dramatically fewer than that of cells with the NC transfected and non-transfected cells. Conversely, the miR-148a-3p inhibitor transfected cells showed much more invaded cell (Figure 3B). These results suggest that miR-148a-3p may play an important role in cell migration and invasion of OS.

\section{MiR-148a-3p directly targets and represses PIK3CA expression}

To explore the potential target for miR-148a-3p, miRNA target analysis tools TargetScan and MiRanda were used. These two programs all predicted that there is a miR-148a-3p binding site in the 3'-UTR of PIK3CA (Figure 4A). To understand whether the effect of miR$148 \mathrm{a}-3 \mathrm{p}$ on PIK3CA is specific, we performed a luciferase reporter assay, which showed that miR-148a-3p had an obvious inhibiting effect on luciferase intensity of wildtype PIK3CA 3'UTR nut not mutant PIK3CA 3'UTR Figure 4B. Collectively, these data suggest that miR-148a3 p may inhibit PIK3CA expression by targeting its $3^{\prime}-$ UTR. Then we tested whether miR-148a-3p could inhibit the endogenous PIK3CA protein expression. As predicted, a western blot showed that, at $48 \mathrm{~h}$ after transfection, enforced expression of miR-148a-3p significantly repressed PIK3CA protein expression in MG-63 cells (Figure 4C). By comparison, knockdown miR-148a-3p led to a moderate increase of PIK3CA protein expression in MG-63 cells (Figure 4C). Together, these results provide evidence that PIK3CA is a specific target of miR-148a-3p in OS cells.

\section{PIK3CA is involved in miR-148a-3p inhibitory effect}

To investigate whether PIK3CA is involved in miR-148a-3p inhibitory effect on cell proliferation, cell migration and invasion of OS. Firstly, PIK3CA siRNA was used to downregulate PIK3CA epression in MG63 cells (Figure 5A). As expected, PIK3CA knockdown significantly suppressed cell proliferation (Figure 5B), cell migration and invasion of MG-63 cells (Figure 5C).

\section{DISCUSSION}

Recently, many studies have demonstrated that miRNAs are associated with the pathogenesis and clinical metastasis in OS $[23,24]$. Overexpression of miR-148a$3 p$ reduced proliferation and induced apoptosis in OS cells [25]; miR-148a-3p is frequently downregulated and miR-148a-3p inhibits cell proliferation and epithelial mesenchymal transition (EMT) by regulating ERBB3/ AKT2/c-myc and ERBB3/AKT2/Snail signaling in bladder cancer [26]; miR-148a-3p reduced cell migration, invasion and proliferation by targeting DNA methyltransferase enzyme (DNMT1) and runt-related transcription factor 3 
(RUNX3) in laryngeal squamous cell carcinoma (LSCC) $[27,28]$; circling miR-148a-3p has been reported to serve as tumor biomarker [29-31]. However, the roles of miR$148 \mathrm{a}-3 \mathrm{p}$ in OS has not been reported. In this study, it was found miR-148a-3p was downregulated in OS cells. In addition, we analyzed the expression of miR-148a-3p in OS patients and found that the expression of miR-148a-3p was much lower in OS tissues in comparison with paired adjacent bone tissues. All of these evidences indicated that miR-148a-3p might play a significant part in the development of OS. Furthermore, ectopic expression of miR-148a-3p significantly inhibited OS cell proliferation, migration and invasion. These findings suggested that miR-148a-3p might act as a tumor suppressor gene whose downregulation may contribute to the progression and metastasis of OS.

PIK3CA is known to be an oncogene component of PI3K signaling pathway and is implicated in cell proliferation and cancer carcinogenesis [32, 33]. Recently, many miRNAs have been reported to regulate cancer carcinogenesis by targeting PIK3CA, including miR-19a [34], miR-134 [35]; miR-203 [36], miR-375 [22, 37]; miR-422a [38], miR-490-5p [39], et al. In this study, PIK3CA was found as a direct target of miR-148a$3 p$ by bioinformatics analysis, luciferase reporter assay and western blot. Moreover, PIK3CA were involved in inhibitory effects of miR-148a-3p on cell proliferation, migration and invasion in OS. These results indicated that miR-148a-3p might function as a tumor suppressor in OS partly by repressing PIK3CA expression.

In the conclusion, we determined that miR-148a-3p function as a tumor suppressor in OS through targeting PIK3CA. Consequently, these findings provided a molecular basis for the role of miR-148a-3p/PIK3CA in human OS progression and suggested that miR-148a-3p could be a potential target for OS treatment.
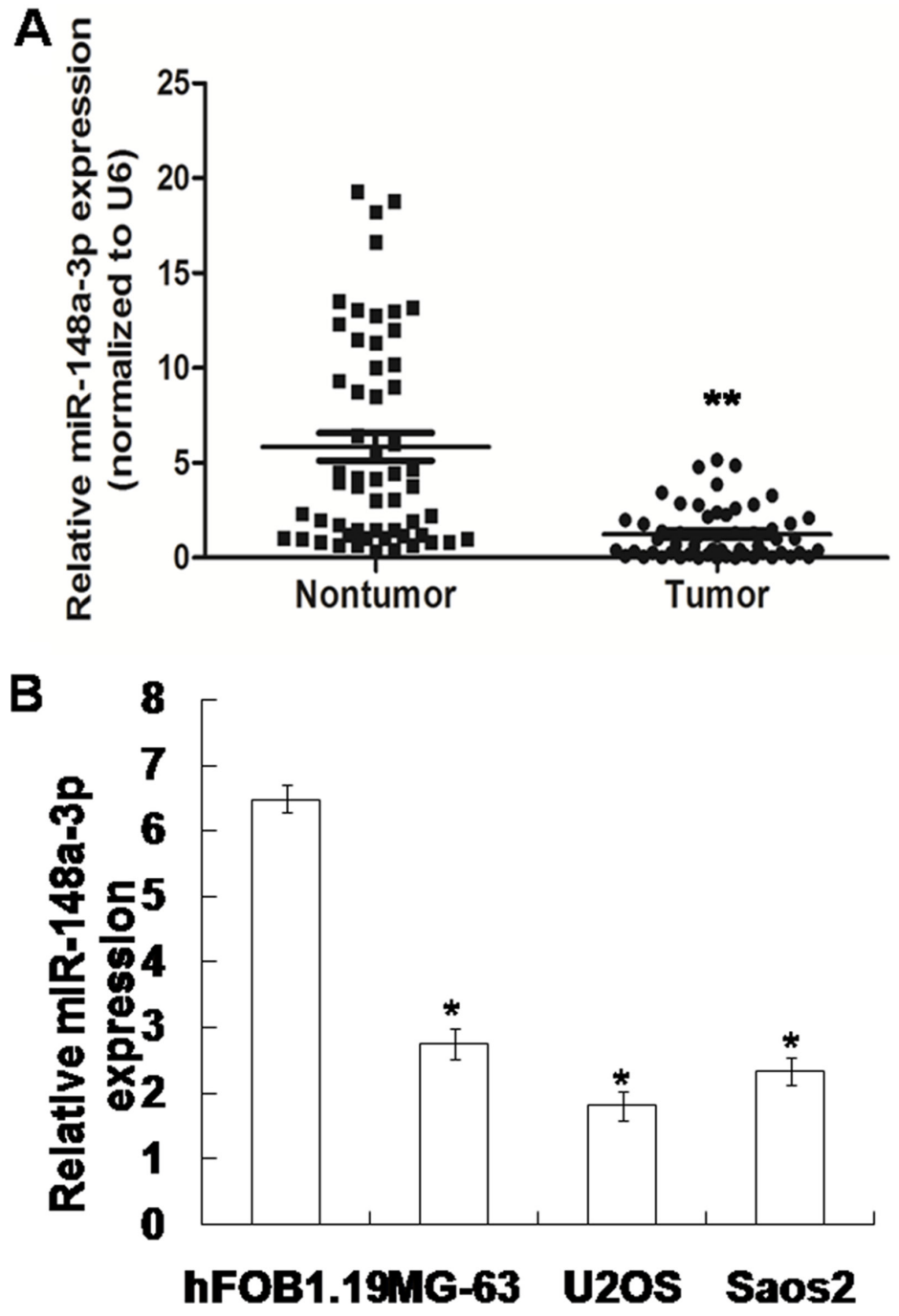

Figure 1: miR-148a-3p is down-regulated in OS. (A) The relative expression of miR-148a-3p was determined in human osteoblast cell line (hFOB1.19) and OS cells (MG-63 and U2OS) by qRT-PCR. (B) The relative expression of miR-148a-3p was determined in 56 pairs OS tissues and the corresponding adjacent non-tumor tissues by qRT-PCR. The expression of miR-148a-3p was normalized to U6. ${ }^{*} P<0.05,{ }^{* *} P<0.01$. 


\section{MATERIALS AND METHODS}

\section{Cell culture and human tissue samples}

The human embryonic kidney-derived cell line HEK293T and human OS cell lines (MG-63, U2OS and Saos2) and human osteoblast cell line hFOB1.19 were maintained in Dulbecco's modified eagle's medium (DMEM, Hyclone, Thermo Fisher Scientific, Waltham, MA, USA) supplemented with $10 \%$ fetal bovine serum (FBS) (GIBCO, NY, USA) and streptomycin $(100 \mathrm{mg} / \mathrm{ml})$, penicillin $(100 \mathrm{U} / \mathrm{ml})$. Cultures were incubated at $37^{\circ} \mathrm{C}$ with $5 \% \mathrm{CO}_{2}$ in a humidified incubator.

Fifty six pairs of human OS tissue and adjacent non-neoplastic tissue samples were obtained immediately after resection from patients undergoing primary surgical treatment in Henan Provincial Luoyang OrthopedicTraumatological Hospital, Luo Yang, China. None of the patients received preoperative chemotherapy or radiotherapy before surgery. Clinical characteristics of patients were detailed in Table 1.The tissue samples were frozen in liquid nitrogen and stored at $-80^{\circ} \mathrm{C}$ until use. Written consent for research purposes was obtained from the patients before tissue collection and the protocol was approved by the Institutional Review Board of Henan Provincial Luoyang Orthopedic-Traumatological Hospital.

\section{Transfection}

Cells were transfected with $20 \mathrm{nmol} / \mathrm{L}$ of miR-148a$3 p$ mimic, miR-148a-3p inhibitor or nonrelative control (NC) duplex (GenePharma, Shanghai, China) and PIK3CA siRNAs or NC siRNA (Ribobo, Guangzhou, China) using FuGene HD transfection reagent (Roche, Indianapolis, IN, USA) according to the manufacturer's protocol.

\section{RNA extraction and quantitative real-time RT- PCR analysis}

Total RNA was extracted from human tissues or cell lines using TRIzol reagent (Invitrogen Life Technologies, Carlsbad, CA, USA) according to the manufacturer's protocol. For miRNA detection, $2 \mu \mathrm{g}$ of small RNA was reverse transcribed to cDNA using miRNA FirstStrand cDNA Synthesis kit (Invitrogen) according to the manufacturer's instructions. Quantitative real-time PCR (qRT-PCR) analysis for miR-148a-3p was performed in triplicate with TaqMan MicroRNA Assay (Applied
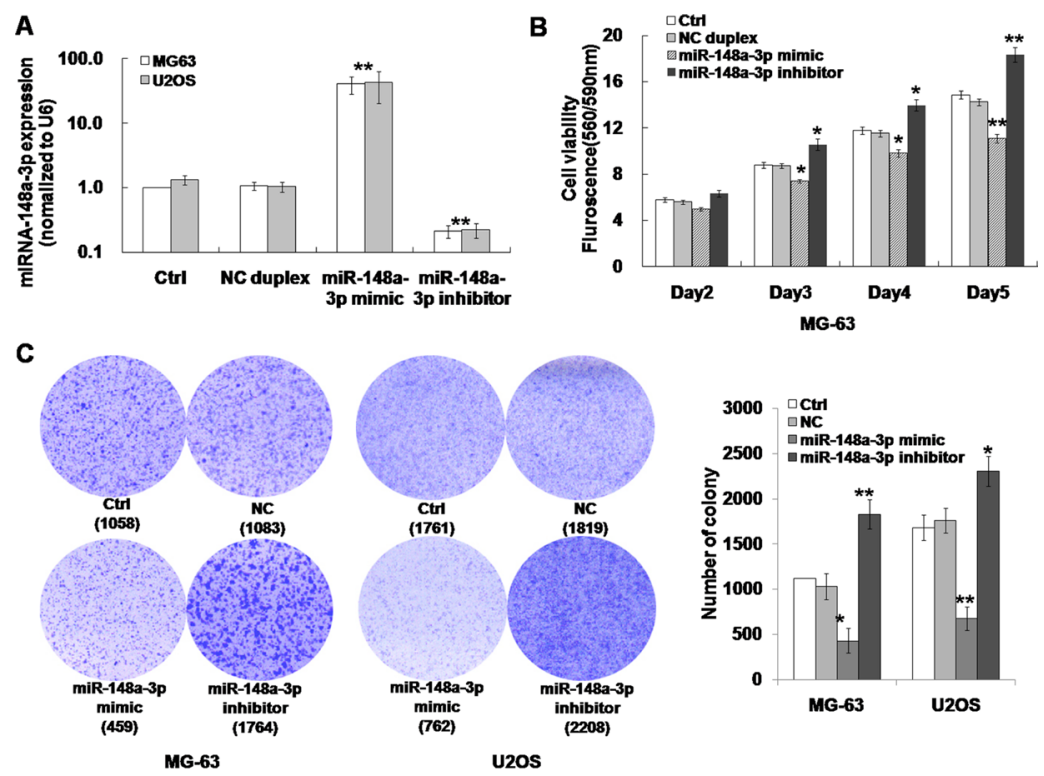

D

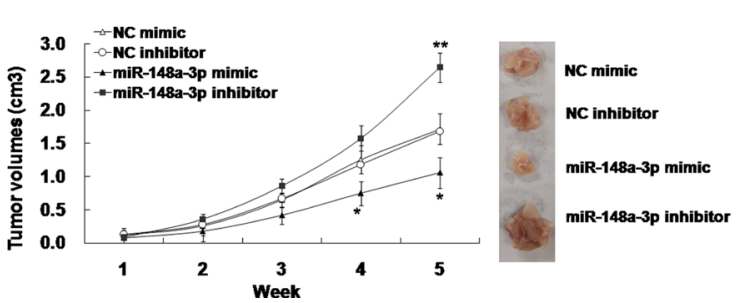

Figure 2: miR-148a-3p inhibits cell proliferation in OS cells and tumorigenicity in nude mice. (A) The relative expression of miR-148a-3p was determined in human OS cells (MG-63 and U2OS). (B) Cell viability was determined in MG-63 (Left panel) and U2OS (Right panel) cells. (C) Colony formation assay was determined in MG-63 and U2OS cells. (D) The effect of miR-148a-3p on the tumor growth in xenograft nude mice. Representative results (B-D) in cells transfected with miR-148a-3p mimic, miR-148a-3p inhibitor or NC duplex. The data were represented by mean \pm SD. ${ }^{*} P<0.05,{ }^{* *} P<0.01$. 
Table 1: The data of patients $(n=56)$

\begin{tabular}{lc}
\hline Variable & Value \\
\hline Age (years) & $20.6 \pm 8.4$ \\
Gender (male) & $32(57.1 \%)$ \\
Tumor size* $(\mathrm{cm})$ & 7.59 \\
Tumor grade & 26 \\
$\quad$ High & 30 \\
$\quad$ Low & 30 \\
Tumor stage** & 18 \\
I & 23 \\
II & 15 \\
\hline
\end{tabular}

*Diameter of the biggest nodule

Biosystems) according to the manufacturer's instructions. Small nuclear U6 snRNA was used as an internal control. To detect the target genes, $2 \mu \mathrm{g}$ of total RNA was reverse transcribed to cDNA using oligo (dT) primers and Moloney murine leukemia virus reverse transcriptase (Promega). GAPDH were used to normalize expression. Data analysis was performed using the $2^{-\Delta \Delta \mathrm{Ct}}$ method.

\section{Cell viability and colony formation assays}

Cell proliferation was analyzed by Cell Titer-Blue cell viability assay (Promega Corporation, Madison, WI, USA) according to the manufacturer's instructions, and the fluorescence ratios were recorded using a multi-plate reader (Synergy 2, BioTek, Winooski, VT, USA). Cell viability was determined at 1, 2, 3, 4 and 5 days after transfection. In addition, 2000 transfected cells were seeded in fresh 6-well plates in triplicate and maintained in DMEM containing 10\% FBS for 2 weeks. Next, cell colonies were fixed in $20 \%$ methanol and stained with $0.1 \%$ coomassie brilliant blue R250 at room temperature for $15 \mathrm{~min}$. Finally, the colonies were counted using an ELIspot Bioreader 5000 (BIO-SYS, Karben, GE).

\section{Cell migration and invasion assay}

For the cell migration assay, $2 \times 10^{5}$ transfected MG-63 and U2OS cells in serum-free media were seeded in the upper chamber of transwell units (Corning, NY, USA) with $8 \mu \mathrm{m}$ pore size polycarbonate filter under serum free condition. The lower chamber was filled with $500 \mu \mathrm{L}$ DMEM containing $10 \%$ FBS. After incubation for $24 \mathrm{~h}$,

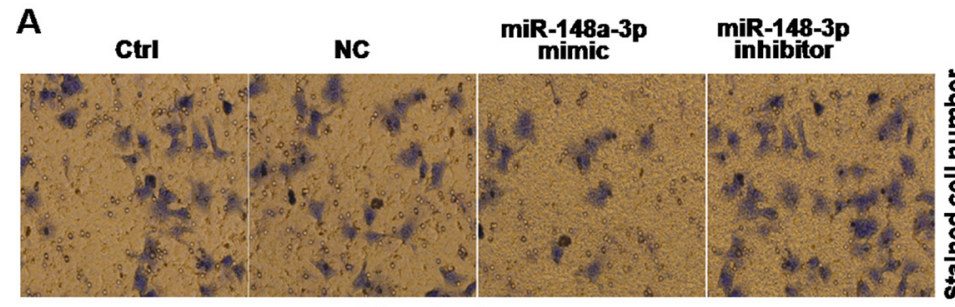

Migration Assay

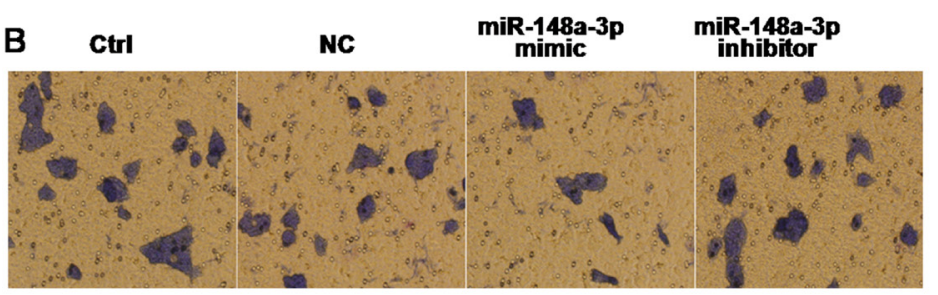

Invasion Assay
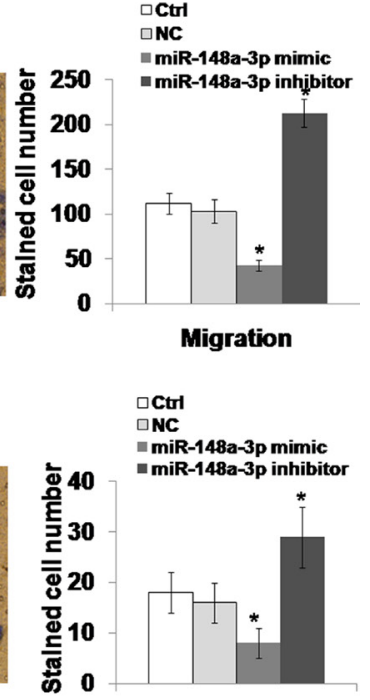

Invasion

Figure 3: miR-148a-3p inhibits cell migration and invasion in OS cells. (A) Cell migration assay was determined in MG-63 cells. (B) Cell invasion assay was determined in MG-63 cells. Cells were transfected with miR-148a-3p mimic, miR-148a-3p inhibitor or NC duplex. Left panel was the representative data of assay; Right panel was the summarized data of assay. The data were represented by mean $\pm \mathrm{SD} .{ }^{*} P<0.05,{ }^{* *} P<0.01$. 
cells on the upper surface of the filter were completely removed by wiping with a cotton swab. Then the filters were fixed with $4 \%$ paraformaldehyde and stained with $0.1 \%$ coomassie brilliant blue R250 for $20 \mathrm{~min}$. The number of cells that migrated through the pores to the lower surface of the filter was counted and analyzed with a digital microscope system (IX81; Olympus). Triplicate samples were acquired and the data were expressed as

\section{A Position 43-49 of PIK3CA3' UTR 5' ... CuCUgGaUduCCACACUgCACUgu... hsa-miR-148a-3p $\quad 3^{\prime} \quad$ UgUUUCAAGACAUCACGUGACU}

PIK3CA3' UTR-Mut 5 ' ... CUCUGGAAagCACACACGUGACU...

B

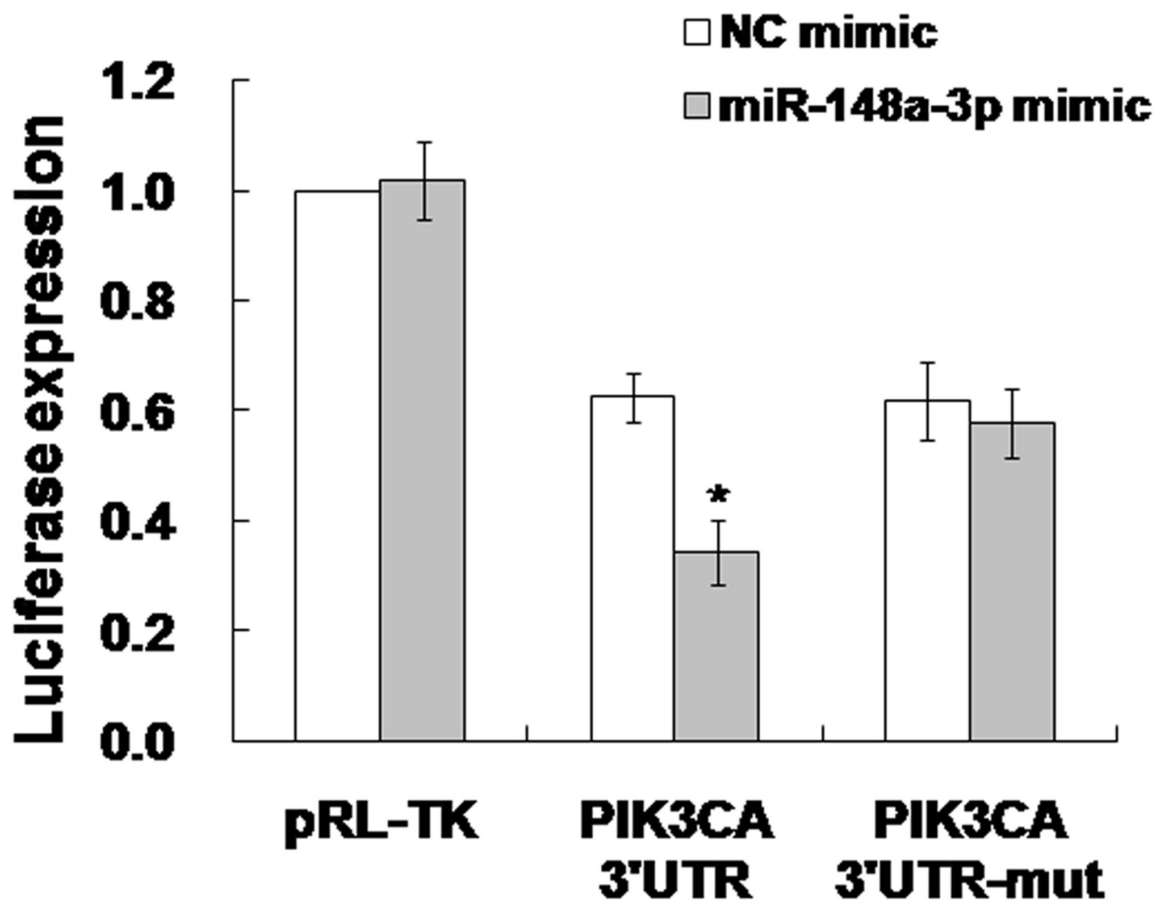

$\mathbf{C}$

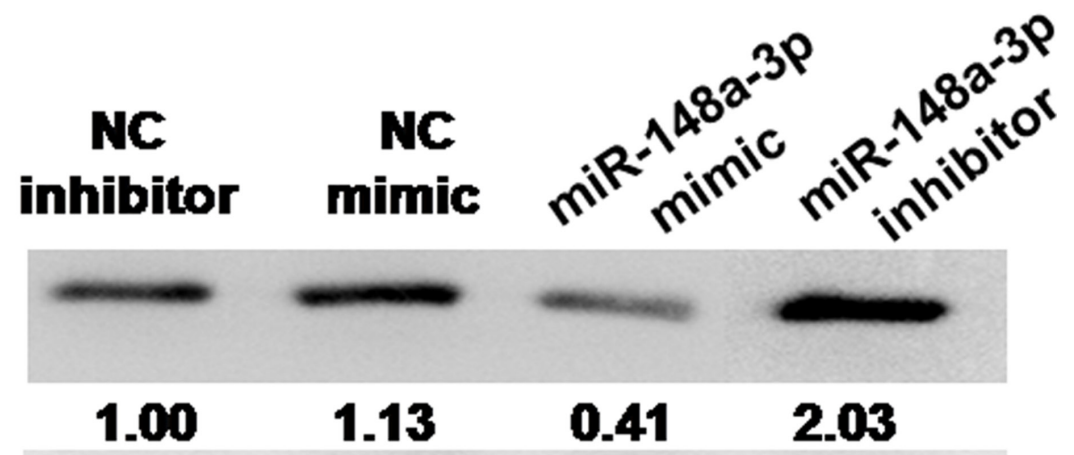

GAPDH

Figure 4: miR-148a-3p directly targets PIK3CA. (A) The predicted miR-148a-3p binding site within the PIK3CA 3'-UTR and a mutated version generated by site directed mutagenesis are shown. (B) Luciferase reporter assay illustrating direct binding of miR-148a-3p to the WT, but not mutant sequences within the 3'-UTR of PIK3CA. (C) The protein expression of PIK3CA was regulated by miR-148a-3p in MG-63 cells. GAPDH was used as a control. The data were represented by mean \pm SD. ${ }^{*} P<0.05,{ }^{* *} P<0.01$. 
A

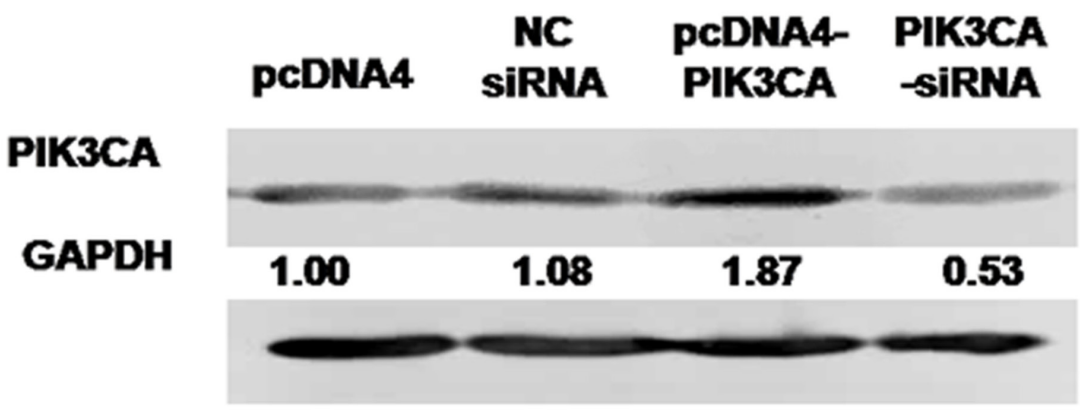

B
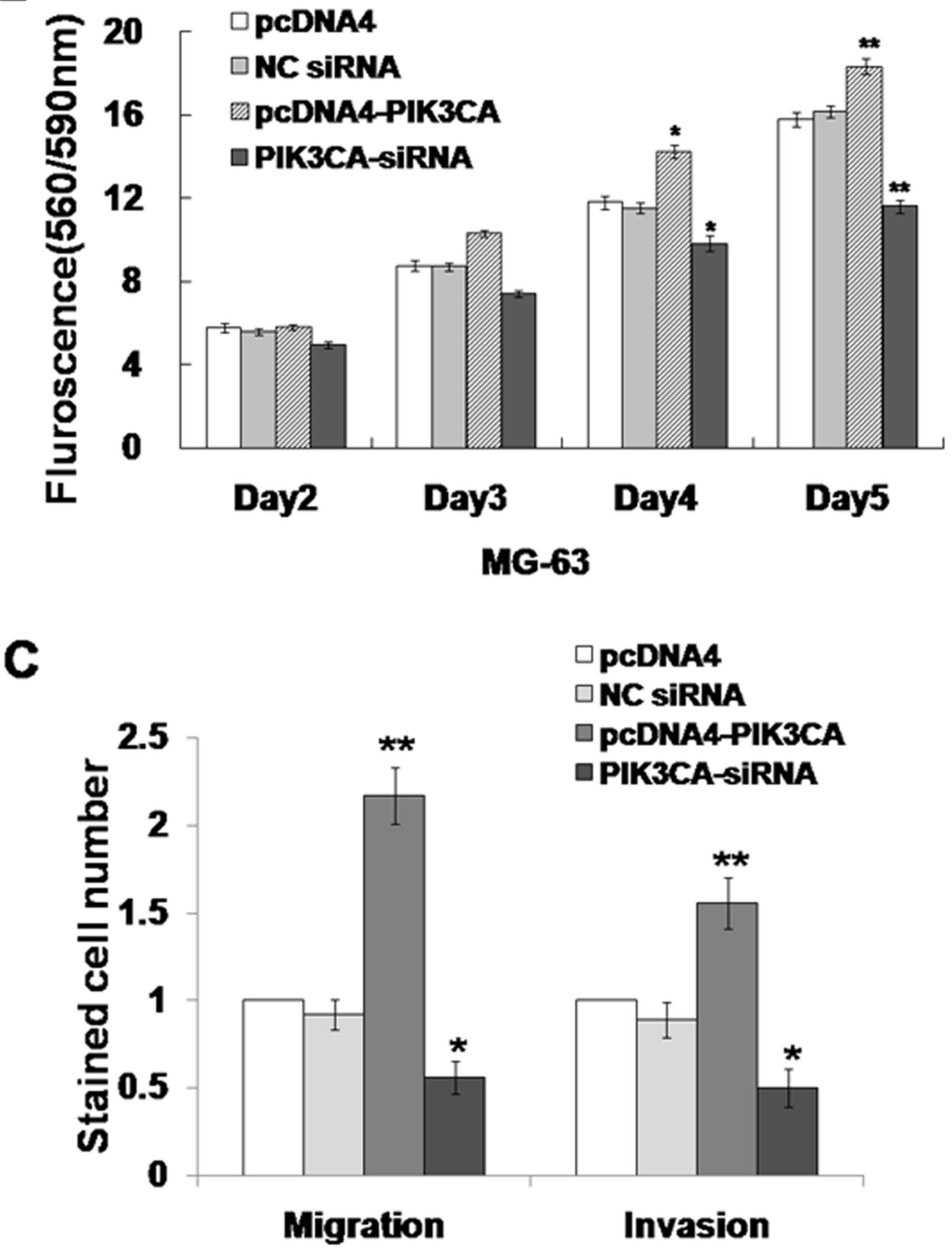

Figure 5: PIK3CA is involved in miR-148a-3p inhibited cell proliferation, migration and invasion in MG-63 cells. (A) The expression of PIK3CA in MG-63 cells transfected with pcDNA4-PIK3CA, PIK3CA siRNA or pcDNA4, NC siRNA. (B) Downregulation of PIK3CA suppressed cell proliferation in MG-63 cells. (C) Down-regulation of PIK3CA suppressed cell migration and invasion in MG-63 cells. The data were represented by mean \pm SD. ${ }^{*} P<0.05,{ }^{* *} P<0.01$. 
the average cell number of 5 fields. For the cell invasion assay, the similar protocol of cell migration assay was used except that the transwell units were pre-coated with $200 \mu \mathrm{g} / \mathrm{ml}$ Matrigel (BD Biosciences, San Jose, CA, USA) and incubated overnight. Cells that had invaded the Matrigel and reached the lower surface of the filter were counted.

\section{Tumorigenicity assays in nude mice}

Male BALB/c nude mice (5 to $6 \mathrm{wk}$ of age) were obtained from Henan Provincial Experimental Animal Center (Luoyang, China). Animal handling and experimental procedures were approved by the Animal Experiments Ethics Committee of Henan Provincial Luoyang Orthopedic-Traumatological Hospital. For in vivo tumorigenicity assay, all pyrimidine nucleotides in the miR-148a-3p mimic, miR-148a-3p inhibitor or NC duplex were substituted by their 2'-O-methyl analogues to improve RNA stability. MiR-148a-3p mimic or miR148a-3p inhibitor transfected MG-63 cells $\left(1 \times 10^{6}\right)$ were suspended in $100 \mathrm{uL}$ PBS and then injected s.c. into left side of the posterior flank of $6 \mathrm{BALB} / \mathrm{c}$ nude mice, respectively. NC transfected or nontransfected MG-63 cells $\left(1 \times 10^{6}\right)$ were injected subcutaneously into right side of same 12 mice. Tumor growth was examined daily and the tumor volumes were calculated every week using the formula for hemi-ellipsoids: $\mathrm{V}=$ length $(\mathrm{cm})$ $\times$ width $(\mathrm{cm}) \times$ height $(\mathrm{cm}) \times 0.5236$. After 5 weeks, the mice were sacrificed and the tumors were dissected and photographed.

\section{Western blotting analysis}

Proteins were extracted using a modified RIPA buffer with $0.5 \%$ sodium dodecyl sulfate (SDS) and the proteinase inhibitor cocktail (Complete Mini, Roche). Equal amounts of protein were separated on $10 \%$ SDSPAGE gel, and then transferred to a PVDF membrane (Immobilon P-SQ, Millipore, Billerica, MA, USA). After blocked with $5 \%$ non-fat dried milk, the membrane was incubated with anti-PIK3CA (Abcam, England) at 1:1000 dilution and anti-GAPDH antibody (Proteintech, Chicago, USA) at 1:20,000 dilution. After washing with TBST (10 $\mathrm{mM}$ Tris, $\mathrm{pH} 8.0,150 \mathrm{mM} \mathrm{NaCl}$, and $0.1 \%$ Tween 20), the membranes were incubated with HRP-conjugated goat anti-rabbit antibody or goat anti-mouse antibody (1:10000 dilution, KPL, Gaithersburg, MA,USA) for $2 \mathrm{~h}$ at room temperature. Proteins bands were visualized using ECL reagents (Pierce, Rockford, IL, USA).

\section{Luciferase reporter assay}

The 3'-UTR fragment of PIK3CA (Genbank accession no. NM_006218.3) containing the putative binding sequence was amplified using the primers 5'-
AAAGATAACTGAGAAAATGAAAGCTC-3' (forward) and 5'-GAAGAAAGCTGACCATGCTGCTATG -3' (reverse). The resulting PCR product was cloned into a firefly luciferase reporter vector (pGL3; Promega Corporation, Madison, WI, USA), and termed pGL3PIK3CA-3'UTR. A plasmid that carried mutations in the complementary sites for the seed region of miR-148a-3p was generated based on the pGL3-PIK3CA-3'UTR plasmid using a MutanBEST Kit (Takara Bio Inc., Shiga, JP), and termed pGL3-PIK3CA-3'UTR-mut. The correctness of the plasmids was confirmed by sequence analysis. HEK293T cells were co-transfected pGL3-PIK3CA-3'UTR or pGL3PIK3CA-3'UTR-mut and the miR-148a-3p mimic or NC duplex. The cells were collected $48 \mathrm{hrs}$ after transfection, and were analyzed for luciferase activity using the dualluciferase reporter assay kit (Promega Corporation). The pRL-TK construct (Promega Corporation, Madison, WI, USA) was also transfected as a normalization control.

\section{miRNA target prediction}

TargetScan (http://www.targetscan.org) and MiRanda (http://www.microrna.org/microrna/home.do) was used to predict miRNA targets and conserved sites bound by the seed region of miR-148a-3p.

\section{Statistical analysis}

Data were presented as the means \pm standard deviation of at least three experiments. Statistical analysis was performed using SPSS 17.0. A one-way analysis of variance (ANOVA) test, least significant difference (LSD) test, Chisquare test and Student's t test were used for statistical analysis.

\section{CONFLICTS OF INTEREST}

None.

\section{FUNDING}

This study was supported by Henan Luoyang Technology Program Health Project (1603004A-10).

\section{REFERENCES}

1. Mirabello L, Troisi RJ, Savage SA. OS incidence and survival rates from 1973 to 2004: data from the surveillance, epidemiology,and end results program. Cancer. 2009; 115:1531-43.

2. Cho Y, Jung GH, Chung SH, Kim JY, Choi Y, Kim JD. Long-term survivals of stage IIb OS: a 20-year experience in a single institution. Clin Orthop Surg. 2011; 3:48-54.

3. Lewis VO. What's new in musculoskeletal oncology. J Bone Joint Surg Am. 2009; 91:1546-1556. 
4. Zhou W, Hao M, Du X, Chen K, Wang G, Yang J. Advances in targeted therapy for OS. Discov Med. 2014; 17:301-7.

5. Sampson VB, Yoo S, Kumar A, Vetter NS, Kolb EA. MicroRNAs and Potential Targets in OS: Review. Front Pediatr. 2015; 3:69.

6. Jones KB, Salah Z, Del Mare S, Galasso M, Gaudio E, Nuovo GJ, Lovat F, LeBlanc K, Palatini J, Randall RL, Volinia S, Stein GS, Croce CM, et al. miRNA signatures associate with pathogenesis and progression of OS. Cancer Res. 2012; 72:1865-77.

7. Chang L, Shrestha S, LaChaud G, Scott MA, James AW. Review of microRNA in OS and chondrosarcoma. Med Oncol. 2015; 32:613. d

8. Van Wynsberghe PM, Chan SP, Slack FJ, Pasquinelli AE. Analysis of microRNA expression and function. Method Cell Biol 2011; 106:219-252.

9. Tang JT, Fang JY. MicroRNA regulatory network in human colorectal cancer. Mini Rev Med Chem. 2009; 9:921-926.

10. Suzuki H, Maruyama R, Yamamoto E, Kai M. DNA methylation and microRNA dysregulation in cancer. Mol Oncol. 2012; 6:567-578.

11. Schotte D, Pieters R, Den Boer M. MicroRNAs in acute leukemia: from biological players to clinical contributors. Leukemia. 2012; 26:1-12.

12. Sandoval J, Esteller M. cancer epigenomics. beyond genomics. Curr Opin Genet Dev. 2012; 22:50-55.

13. Rottiers V, Najafi-Shoushtari SH, Kristo F, Gurumurthy S, Zhong L, Li Y, Cohen DE, Gerszten RE, Bardeesy N, Mostoslavsky R. MicroRNAs in Metabolism and Metabolic Diseases. In Cold Spring Harbor Symposia on Quantitative Biology. Cold Spring Harbor Laboratory Press. 2011. pp. 225-233.

14. Song B, Ju J. Impact of miRNAs in gastrointestinal cancer diagnosis and prognosis. Expert Rev Mol Med. 2010; 12:e33.

15. Tian X, Zhang J, Yan L, Dong JM, Guo Q. MiRNA-15a inhibits proliferation, migration and invasion by targeting TNFAIP1 in human OS cells. Int J Clin Exp Pathol. 2015; 8:6442-9.

16. Jin J, Cai L, Liu ZM, Zhou XS. miRNA-218 inhibits OS cell migration and invasion by down-regulating of TIAM1, MMP2 and MMP9. Asian Pac J Cancer Prev. 2013; 14:3681-4.

17. Cheng DD, Yu T, Hu T, Yao M, Fan CY, Yang QC. MiR$542-5 p$ is a negative prognostic factor and promotes OS tumorigenesis by targeting HUWE1. Oncotarget. 2015; 6:42761-72. https://doi.org/10.18632/oncotarget.6199.

18. Chen J, Zhou J, Chen X, Yang B, Wang D, Yang P, He X, Li H. miRNA-449a is downregulated in OS and promotes cell apoptosis by targeting BCL2. Tumour Biol. 2015; 36:8221-9.

19. Jin H, Wang W. MicroRNA-539 suppresses OS cell invasion and migration in vitro and targeting Matrix metallopeptidase-8.Int J Clin Exp Pathol. 2015 Jul 1; 8:8075-82.
20. Sun XH, Geng XL, Zhang J, Zhang C. miRNA-646 suppresses OS cell metastasis by downregulating fibroblast growth factor 2 (FGF2). Tumour Biol. 2015; 36:2127-34.

21. Yang D, Liu G, Wang K. miR-203 Acts as a Tumor Suppressor Gene in OS by Regulating RAB22A. PLoS One. 2015; 10:e0132225.

22. Shi ZC, Chu XR, Wu YG, Wu JH, Lu CW, Lü RX, Ding MC, Mao NF. MicroRNA-375 functions as a tumor suppressor in OS by targeting PIK3CA.Tumour Biol. 2015; 36:8579-84.

23. Miao J, Wu S, Peng Z, Tania M, Zhang C. MicroRNAs in OS: diagnostic and therapeutic aspects. Tumour Biol. 2013 Aug; 34:2093-8.

24. Ram Kumar RM, Boro A, Fuchs B. Involvement and Clinical Aspects of MicroRNA in OS. Int J Mol Sci. 2016 Jun 3;17. pii:E877.

25. Bhattacharya S, Chalk AM, Ng AJ, Martin TJ, Zannettino AC, Purton LE, Lu J, Baker EK, Walkley CR. Increased miR-155$5 \mathrm{p}$ and reduced miR-148a-3p contribute to the suppression of OS cell death. Oncogene. 2016; 35:5282-5294.

26. Wang X, Liang Z, Xu X, Li J, Zhu Y, Meng S, Li S, Wang S, Xie B, Ji A, Liu B, Zheng X, Xie L. Cell Death Dis. 2016; 7:e2503. miR-148a-3p represses proliferation and EMT by establishing regulatory circuits between ERBB3/AKT2/cmyc and DNMT1 in bladder cancer.

27. Wu T, Qu L, He G, Tian L, Li L, Zhou H, Jin Q, Ren J, Wang Y, Wang J, Kan X, Liu M, Shen J, et al. Regulation of laryngeal squamous cell cancer progression by the lncRNA H19/miR-148a-3p/DNMT1 axis. Oncotarget. 2016; 7:11553-66. https://doi.org/10.18632/oncotarget.7270.

28. Jili S, Eryong L, Lijuan L, Chao Z. RUNX3 inhibits laryngeal squamous cell carcinoma malignancy under the regulation of miR-148a-3p/DNMT1 axis. Cell Biochem Funct. 2016; 34:597-605.

29. Bellissimo T, Russo E, Ganci F, Vico C, Sacconi A, Longo F, Vitolo D, Anile M, Disio D, Marino M, Blandino G, Venuta F, Fazi F. Circulating miR-21-5p and miR-148a-3p as emerging non-invasive biomarkers in thymic epithelial tumors. Cancer Biol Ther. 2016; 17:79-82.

30. Yan Y, Wang X, Venø MT, Bakholdt V, Sørensen JA, Krogdahl A, Sun Z, Gao S, Kjems J. Circulating miRNAs as biomarkers for oral squamous cell carcinoma recurrence in operated patients. Oncotarget. 2017; 8:8206-8214. https:// doi.org/10.18632/oncotarget.14143.

31. Luo Y, Zhang C, Tang F, Zhao J, Shen C, Wang C, Yu P, Wang M, Li Y, Di JI, Chen R, Rili G. Bioinformatics identification of potentially involved microRNAs in Tibetan with gastric cancer based on microRNA profiling. Cancer Cell Int. 2015; 15:115.

32. Bader AG, Kang S, Zhao L, Vogt PK. Oncogenic PI3K deregulates transcription and translation. Nat Rev Cancer. 2005; 5:921-929.

33. Parsons R. Phosphatidylinositol 3-kinase inhibitor are a triple threat to ovarian cancer. Clin Cancer Res. 2005; 11:7965-7966. 
34. Xiao W, Gao Z, Duan Y, Yuan W, Ke Y. Downregulation of miR-19a exhibits inhibitory effects on metastatic renal cell carcinoma by targeting PIK3CA and inactivating Notch signaling in vitro. Oncol Rep. 2015; 34:739-46.

35. El-Daly SM, Abba ML, Patil N, Allgayer H. miRs-134 and -370 function as tumor suppressors in colorectal cancer by independently suppressing EGFR and PI3K signalling. Sci Rep. 2016; 6:24720.

36. Liang M, Shi B, Liu J, He L, Yi G, Zhou L, Yu G, Zhou $\mathrm{X}$. Downregulation of miR-203 induces overexpression of PIK3CA and predicts poor prognosis of gastric cancer patients. Drug Des Devel Ther. 2015; 9:3607-16.
37. Wang Y, Tang Q, Li M, Jiang S, Wang X. MicroRNA-375 inhibits colorectal cancer growth by targeting PIK3CA. Biochem Biophys Res Commun. 2014; 444:199-204.

38. Liang H, Wang R, Jin Y, Li J, Zhang S. MiR-422a acts as a tumor suppressor in glioblastoma by targeting PIK3CA. Am J Cancer Res. 2016; 6:1695-707. eCollection 2016.

39. Chen K, Zeng J, Tang K, Xiao H, Hu J, Huang C, Yao W, Yu G, Xiao W, Guan W, Guo X, Xu H, Ye Z. miR-490-5p suppresses tumour growth in renal cell carcinoma through targeting PIK3CA. Biol Cell. 2016; 108:41-50. 